\title{
CONIZACIÓN LEEP EN NIE DE ALTO GRADO EN UNA INSTITUCIÓN PRIVADA DE SALUD
}

\author{
Eutimio Roa B., Eduardo Osorio F., Sergio Lizana C., Ramiro Herrera Z.
}

Unidad de Patología del Tracto Genital Inferior, Clínica Dávila.

\section{RESUMEN}

Antecedentes: La conización cervical del cuello uterino es considerada el tratamiento de elección de las neoplasias intraepiteliales de alto grado. Objetivo: Analizar los resultados del tratamiento de esta patología mediante conización Leep. Método: Se revisan los antecedentes y biopsias de 145 pacientes con diagnóstico histológico de neoplasia intraepitelial II y III tratadas con conización cervical por asa Leep en la Unidad de Patología del Tracto Genital Inferior de Clínica Dávila desde el 1 de junio de 2002 al 31 de mayo de 2008. Resultados: Hubo una correlación colpo-biópsica de $80 \%$ (116/145) para Lie de alto grado. Hubo borde positivo en 75 pacientes $(51,7 \%)$ y negativo en $70(48,3 \%)$. Fue necesario un segundo tratamiento en 5 pacientes (3,4\%); en 3 casos por recidivas y en 2 por lesiones invasoras. El $89 \%$ permanece en seguimiento, comprobándose que la totalidad de estas ha tenido un tratamiento considerado satisfactorio. Conclusión: Nuestros resultados confirman que las lesiones cervicales de alto grado deben ser tratadas por métodos escisionales preferentemente, siendo la conización con asa Leep el método de elección por su facilidad de realización, bajo costo de insumos y puede ser realizado sin grandes requerimientos de infraestructura.

PALABRAS CLAVE: Cono cervical, neoplasia intraepitelial, lesión de alto grado, asa LEEP

\section{SUMMARY}

Background: The cervical conización of the uterine cervix is considered the treatment of election of high degree intraepithelial neoplasias. Objective: To analyze the results of treatment of this pathology by Leep Conization. Method: The antecedents and biopsies of 145 patients with histological diagnosis of intraepithelial II and III neoplasia, treated with cervix Conization Leep at Lower Genital Tract Unit of Davila Clinic from June first 2002 to May 312008 are reviewed. Results: There was a colpo-biopsy correlation of $80 \%$ $(116 / 145)$ for high degree CIN. There was positive edge in 75 patients (51.7\%) and negative in $70(48.3 \%)$. A second treatment in 5 patients were necessary (3.4\%); in 3 cases by recurrences and 2 by invasive lesions. $89 \%$ remain in control, verifying itself that the totality of these has had a satisfactory considered treatment. Conclusion: Our results confirm that the high degree cervical lesions must preferably be treated by excision methods, being the Leep Conization the method of election by their facility of accomplishment, low cost and can be made without great infrastructure requirements.

KEY WORDS: Cervical conization, CIN, HSIL, LEEP 


\section{INTRODUCCIÓN}

Es sabida la importancia de pesquisar y tratar las lesiones preinvasoras del cuello uterino con el objetivo de reducir la incidencia del cáncer. La historia natural y los conocimientos actuales de la enfermedad nos permiten saber de la importancia del tipo viral y la persistencia de la infección que afecta el epitelio cervical y la evolución hacia el cáncer.

A lo largo del tiempo se han probado distintos tratamientos y protocolos con el objeto de obtener los mejores resultados en el control de esta patología; entre estos están los métodos ablativos que destruyen la lesión in situ y los escisionales que la extirpan. En base a las observaciones clínicas y los resultados obtenidos, se ha ido acrecentando la evidencia de que los métodos preferentes para las lesiones de bajo grado de malignidad son los ablativos y se prefiere los escisionales para las de alto grado (1). Estos últimos corresponden a la llamada conización cervical en todas sus formas (fría o quirúrgica, láser, radiofrecuencia o leep).

La conización cervical del cuello uterino es considerada el tratamiento de elección de las neoplasias intraepiteliales de alto grado $(2,3)$. Desde su introducción a la práctica clínica, la cirugía de radiofrecuencia (Loop Electrosurgical Escisión Procedure) o conización LEEP, se ha ubicado como procedimiento de elección para dicha intervención, por su simplicidad de ejecución, el carácter de ambulatoria, su bajo costo, las escasas complicaciones y los buenos resultados del corto y largo plazo para el control de estas lesiones $(4,5)$.

El sistema público de salud ha desarrollado una eficiente estructura física y profesional para pesquisar y tratar estas pacientes, lo que no ha sido adoptado en la práctica privada institucional. Clínica Dávila, institución privada de salud, consciente de esta diferencia, ha implementado una Unidad de Patología del Tracto Genital Inferior con el objetivo de concentrar los casos de lesiones precursoras y de cáncer cérvico-uterino.

El objetivo de esta comunicación es presentar nuestra experiencia en el tratamiento de las lesiones de alto grado del cuello uterino mediante conización Leep.

\section{PACIENTES Y MÉTODO}

Se revisan los antecedentes de 145 pacientes, a quienes se realizó conización cervical con asa Leep, por el diagnóstico histológico de neoplasia intraepitelial (Nie) de alto grado de malignidad (Nie II y Nie III) en la Unidad de Patología del Tracto Genital Inferior de Clínica Dávila, en el período com- prendido entre 1 de junio de 2002 al 31 de mayo de 2008.

La paridad del grupo correspondió a 20 nulíparas, 121 multíparas de 1 a 3 y solo 4 mujeres con 4 o más partos. La edad promedio fue de 36 años (rango: 20 a 67 años).

El equipo utilizado es una unidad electroquirúgica Cooper Surgical 2000. Se realizaron tres resecciones con modo blend o cut a 40 watts de labio anterior, posterior y canal endocervical.

La técnica de operación fue la siguiente:

Paciente en posición de litotomía. Pincelado de genitales externos con povidona yodada y colocación de campos estériles, limpieza de vagina con tórula de gasa, pincelado del cuello uterino con solución de lugol para demarcación de las zonas alteradas, y anestesia cervical radiada con mepivacaina al $2 \%$. Resección de labio posterior y anterior con asa de 18 o $20 \mathrm{~mm}$ por 15 o $20 \mathrm{~mm}$, con voltaje de 40 watts en modo blend o cut. Resección de canal cervical con asa cuadrada de $10 \times 10 \mathrm{~mm}$. Cauterización del lecho estromal con electrodo de bola con voltaje de 60 watts hasta cubrir completamente la zona resecada y aplicación de subsulfato férrico (solución de Monsell) sobre la escara para control del sangrado local.

\section{RESULTADOS}

La Tabla I muestra los resultados de exámenes de Papanicolaou (Pap) inicial y colposcopia de diagnóstico de las pacientes. En relación al Pap: 27 $(18,6 \%)$ atípicos, $21(14,5 \%)$ de lesión intraepitelial (Lie) de bajo grado (HPV/Nie I), $93(64,1 \%)$ de Lie de alto grado (Nie II y III), y 4 de cáncer invasor. En relación al diagnóstico colposcópico: 5 (3,4\%) de aspecto benigno, 24 (16,6\%) sugerentes de lesión de bajo grado, $116(80 \%)$ de alto grado y ninguno sugerentes de cáncer invasor. Esto establece una correlación colpobiópsica de 80\% (116/145) para Lie de alto grado.

Tabla I

PAP Y COLPOSCOPIA DE INGRESO ( $n$ : 145)

\begin{tabular}{lcccc}
\hline & $\begin{array}{r}\text { Atípico } \\
\mathrm{n}(\%)\end{array}$ & $\begin{array}{r}\text { Lie BG } \\
\mathrm{n}(\%)\end{array}$ & $\begin{array}{r}\text { Lie AG } \\
\mathrm{n}(\%)\end{array}$ & $\begin{array}{c}\mathrm{Ca} \\
\mathrm{n}(\%)\end{array}$ \\
\hline Papanicolaou & $27(18,6)$ & $21(14,5)$ & $93(64,1)$ & $4(2,8)$ \\
Colposcopia & $5(3,4)$ & $24(16,6)$ & $116(80,0)$ & $0(0,0)$ \\
\hline
\end{tabular}

Lie: lesión intraepitelial. BG: bajo grado. AG: alto grado. Ca: cáncer invasor. 
En la Tabla II presentamos la concordancia histológica entre la biopsia previa y el resultado histológico definitivo, con un 70,6\% para los casos de Nie II y de $85,7 \%$ para Nie III (global $77,9 \%$ ). Hubo 3 casos de microinvasión y 1 de invasión que corresponde a $2,8 \%$.

\section{Tabla II \\ CORRELACION HISTOLÓGICA ENTRE BIOPSIA COLPOSCÓPICA E HISTOLOGÍA FINAL ( $n: 145)$}

\begin{tabular}{lccccc}
\hline Biopsia & $\mathrm{n}$ & $\begin{array}{c}\text { Norm } \\
\mathrm{n}(\%)\end{array}$ & $\begin{array}{r}\text { Lie BG } \\
\mathrm{n}(\%)\end{array}$ & $\begin{array}{r}\text { Lie AG } \\
\mathrm{n}(\%)\end{array}$ & $\begin{array}{r}\text { Ca } \\
\mathrm{n}(\%)\end{array}$ \\
\hline Nie II & 75 & $5(6,7)$ & $17(22,7)$ & $53(70,6)$ & $0(0,0)$ \\
Nie III & 70 & $3(4,3)$ & $3(4,3)$ & $60(85,7)$ & $4(5,7)$ \\
Total & 145 & $8(5,5)$ & $20(13,8)$ & $113(77,9)$ & $4(2,8)$ \\
\hline
\end{tabular}

Nie: neoplasia intraepitelial. Lie: lesión intraepitelial. BG: bajo grado. AG: alto grado. Ca: cáncer invasor.

En el compromiso de borde quirúrgico se encontró borde positivo en 75 pacientes $(51,7 \%)$ y borde negativo en 70 (48,3\%). Fue necesario un segundo tratamiento en 5 pacientes $(3,4 \%)$; en 3 casos por recidivas y en 2 casos de lesiones invasoras (Tabla III).

Se registraron complicaciones en 4 ocasiones $(2,8 \%)$. Un caso de cervicorragia precoz (ninguna durante la realización del procedimiento), 2 tardías (48 horas a 15 días) y una sinequia cervical.

Las pacientes fueron dadas de alta en buenas condiciones después de un periodo de observación de tres horas en la sección de recuperación postoperatoria de la clínica. Todas fueron controladas dos veces el primer mes y posteriormente a segui- miento citocolposcópico cada cuatro meses por dos años; 129 pacientes están actualmente en control (89\%) con pérdida de seguimiento de $16(11 \%)$. De las 129 pacientes en control mantenido, 113 se han realizado al menos un Pap, 103 con primer Pap normal $(91,2 \%)$, y 10 con Pap alterado $(8,8 \%)$ informados como: 1 atípico, 5 Lie de bajo grado y 4 Lie de alto grado.

Se estima como curación solo a aquellas pacientes con más de un año de seguimiento con citología y colposcopía normal (73/82), que corresponde al $89 \%$ de la población en seguimiento.

\section{DISCUSIÓN}

Nuestra experiencia tabulada muestra los resultados de la Unidad de Patología Cervical de Clínica Dávila, que funciona en forma similar a las de los servicios públicos de salud en cuanto a la derivación de pacientes, por médicos ginecólogos de la institución y de los centros privados de atención relacionados. Esto nos permite concentrar y aplicar guías de tratamiento desarrolladas en la Clínica y aceptadas por el Ministerio de Salud. En la unidad se realiza el diagnóstico y tratamiento ablativos, o quirúrgicos. Todos los casos se analizan en comité oncológico con la presencia de anátomo-patólogos planificándose su tratamiento complementario cuando es necesario. La evaluación clínica y de los exámenes para la etapificación final permite al grupo generar consensos para el seguimiento hasta cumplir los plazos recomendados, y posteriormente remitirlos a sus médicos tratantes.

La edad de las pacientes es similar a la observada en la mayoría de los trabajos publicados, observándose una tendencia a encontrar una mayor proporción de pacientes menores de 40 años con lesiones de alto grado de malignidad $(68,1 \%)$ y un

Tabla III

SEGUNDO TRATAMIENTO POR RECIDIVA

\begin{tabular}{cllll}
\hline Paciente & Histología cono & Recidiva & Segundo tto & Histología \\
\hline 1 & Nie III & NIE II & DC & -- \\
2 & Nie II + HPV & HPV & DC & -- \\
3 & Nie III + HPV & HPV & Histerectomía & Normal \\
4 & Microinvasión & --- & WM & Microinvasión \\
5 & Ca & --- & WM & Normal \\
\hline
\end{tabular}

Nie: neoplasia intraepitelial. HPV: virus papiloma humano. tto: tratamiento. Cáncer invasor. WM: Wertheim-Meigs. DC: diatermo coagulación. 
tercio de ellas menores de 30 años, lo que está en concordancia con el aumento de mujeres que inician actividad sexual a mas temprana edad (6).

La citología de ingreso y colposcopía se correlacionan en porcentajes cercanos al 65 y $80 \%$ respectivamente con el tipo de lesión que tratamos, esto es esperable ya que las citologías provienen de diferentes laboratorios y la colposcopia fue realizada por cuatro operadores. Esto también se observa en la correlación biopsia/histología que alcanza a un $77,9 \%$, similar a lo publicado y con un porcentaje de $2,8 \%$ de lesiones invasoras, cifra también similar a otra casuística internacional (7).

En nuestros casos, el compromiso de bordes alcanzó a un $51,7 \%$. En todo caso esta circunstancia, no reviste un gran riesgo de recidiva de lesiones como ha sido publicado, debido a varias razones entre las cuales, está el hecho de tratar los remanentes de lesión con la cauterización del lecho estromal $(8,9)$.

Durante el seguimiento se observaron solo tres recidivas, una Nie II y dos de afección viral que requirieron de un segundo tratamiento, los otros dos casos fueron histerectomías por lesiones de microinvasión y otra por cáncer invasor. El seguimiento alcanzó a un $89 \%$ de las consultas iniciales. Estas cifras las consideramos aceptables considerando que varias pacientes vuelven con su médico tratante y que probablemente siguen sus controles fuera de la Unidad.

Se observó un alto porcentaje de pacientes en seguimiento con Pap normales al primer control $(91,2 \%)$ lo que está en concordancia con la evolución de las pacientes. Esto se mantiene en el tiempo observando un $97,9 \%$ de pacientes, sin evidencias de recidiva de la enfermedad o que requieran otro tratamiento, muchas de las cuales ya han completado un tiempo superior a los dos años y con el respectivo cierre de los casos.

\section{CONCLUSIONES}

Las lesiones cervicales de alto grado deben ser tratadas por métodos escisionales preferentemente, siendo la conización con asa Leep el método de elección por su facilidad de realización, bajo costo de insumos y que puede ser realizado sin grandes requerimientos de infraestructura. Los resultados de esta clase de tratamiento son buenos, y alcanzan cifras globales cercanas al $90 \%$ al año de seguimiento.

\section{BIBLIOGRAFÍA}

1. Wright TC Jr, Cox JT, Massad LS, Carlson J, Twiggs LB, Wilkinson EJ; American Society for Colposcopy and Cervical Pathology. 2001 consensus guidelines for the management of women with cervical intraepithelial neoplasia. Am J Obstet Gynecol 2003;189(1):295304.

2. Martin-Hirsch PL, Paraskevaidis E, Kitchener H. Surgery for cervical intraepithelial neoplasia. Cochrane Database Syst Rev 2000;(2):CD001318.

3. Alonso I, Torné A, Puig-Tintoré LM, Esteve R, Quinto L, Campo E, Pahisa J, Ordi J. Pre-and post-conization high-risk HPV testing predicts residual/recurrent disease in patients treated for CIN 2-3. Gynecol Oncol 2006;103(2):631-6.

4. Cox JT. Management of cervical intraepithelial neoplasia. Lancet 1999;353(9156):857-9.

5. Ferenczy A, Choukroun D, Arseneau J. Loop electrosurgical excision procedure for squamous intraepithelial lesions of the cervix: advantages and potential pitfalls. Obstet Gynecol 1996;87(3):332-7.

6. Chan KS, Kwok CW, Yu KM, Sin SY, Tang LC. A three-year review of treatment of cervical intraepithelial neoplasia with large loop excision of the transformation zone. Hong Kong Med J 1997;3(1):21-6.

7. Dunn TS, Burke M, Shwayder J. A "see and treat" management for high-grade squamous intraepithelial lesion pap smears. J Low Genit Tract Dis 2003;7(2):1046.

8. Histological incomplete excision of CIN after large loop excision of the transformation zone (LLETZ) merits careful follow up, not retreatment.

Murdoch JB, Morgan PR, Lopes A, Monaghan JM. Br J Obstet Gynaecol. 1992;99(12):990-3.

9. Byrne P, Ogueh O, Wilson J, Sant-Cassia LJ. Outpatient loop diathermy cone biopsy. J Obstet Gynaecol 1993:13:130-4 